\title{
Monitoring the State of Small Power Equipment
}

\author{
Andrei Dragomirescu ${ }^{1, *}$, Crăița Carp-Ciocârdia ${ }^{2}$, Carmen-Anca Safta $^{1}$, Nicolae Orășanu ${ }^{2}$, \\ and Ioan Magheți ${ }^{2}$ \\ ${ }^{1}$ University Politehnica of Bucharest, Department of Hydraulics, Hydraulic Machinery and \\ Environmental Engineering, 313 Splaiul Independentei, Bucharest, Romania \\ ${ }^{2}$ University Politehnica of Bucharest, Department of Mechanics, 313 Splaiul Independentei, Bucharest, \\ Romania
}

\begin{abstract}
The paper presents analyses of vibrations measured on power equipment that can be encountered in rural spaces. A centrifugal pump that can be used in a small supply water network or in an irrigation system and a small hydraulic turbine designed for small hydropower plants were tested. Power spectra obtained with the Fast Fourier Transform for the vibrations measured at the pump and at the turbine are presented and discussed and defects that affect the normal operation of the machines are identified. The results highlight the importance of vibration diagnosis in the process of monitoring the operation of the machines, as a proactive maintenance method.
\end{abstract}

\section{Introduction}

For the last two decades, the Romanian rural economy has been undergoing a continuum process of development aimed at improving the quality of life. Important investments in infrastructure, reflected in new facilities for electric power and thermal energy production, drinking water supplies, public sewerage systems, and waste management, have changed rural areas, which cover $87 \%$ of the country [1]. The Rural Development Programme for Romania, which is available for the period 2014-2020, supports investments in projects related to water efficiency and renewable energy in order to improve the competitiveness of agriculture [2].

Irrigation and water supply systems as well as systems for harvesting the renewable energy of small rivers require specific hydraulic machines, namely pumps and water turbines. The efficiency of these machines depends not only on their design and operating conditions, but also on the maintenance management. Vibration monitoring and analysis are nowadays widely used for the predictive maintenance [3], since they allow to obtain valuable information on the state of different types of equipment. In case of hydraulic machines, such as pumps or water turbines, the vibrations have a significant impact on the safety and reliable operation of these machines [4]. There are various sources of vibrations in hydraulic machines: the complexity

\footnotetext{
${ }^{*}$ Corresponding author: andrei.dragomirescu@upb.ro
} 
of the flows inside the runners, the mechanical and hydraulic imbalances of the rotating parts, the wear of the bearings, the misalignment of the shafts, etc. The complexity of the flow causes turbulence and boundary layer separations that are usually accompanied by pressure fluctuations which are sources of vibrations. The mechanical imbalance can be caused either by added masses, e.g. small debris that can remain stuck between two runner blades or the uneven distribution of the discharge inside the runner, or by subtracted masses, e.g. pieces of material removed by abrasive or cavitation erosion. The hydraulic imbalance is usually caused by the interactions between rotating parts - the runners - and fixed parts - stay vanes, wicket gates, diffuser vanes, or scroll casing tongs. The wear of the bearings occurs naturally, its rate being influenced by the operating regimes, loads, and maintenance. The misalignment of the shafts is usually caused by improper assemblies.

Identifying the causes of vibrations is a complex problem due to the features of the signals in the frequency spectrum, that characterize each particular cause. A frequent mechanical cause of vibrations is the malfunction of the bearings due to their wear that leads to defects.

The present paper presents analyses of the results obtained on two experimental models one of a pumping station, the other of a small hydropower plant. Of all the causes of vibrations highlighted previously, the paper focuses on the identification of those frequencies in the vibrations spectra, that are caused by the defects due to the wear of the bearings of the machines that operate in the investigated installations.

\section{Theoretical considerations regarding the mechanical vibrations caused by the wear of rolling bearings}

Since many machines that have rotating parts are equipped with rolling bearings, an important cause of vibrations are the defects of the bearings. A defective bearing causes excessive vibrations that are transmitted to the casing or to the chassis of the machine, where they can be measured with accelerometers. In this way, a global evaluation of the bearing can be carried out. The deterioration of a bearing is usually a lengthy process that takes place in several stages:

- in the first stage, early minor defects can occur, being indicated by vibrations having ultrasonic frequencies;

- in the second stage, small defects can appear, stimulating the natural frequencies of the bearing elements - these frequencies are usually found in the range 500-2000 Hz;

- in the third stage, as the wear evolves, frequencies corresponding to the defects of the rolling bearings and their harmonics appear, being also possible to identify side bands around them - these frequencies are usually below $500 \mathrm{~Hz}$;

- in the fourth stage, the deterioration becomes significant and leads to an increase in the clearings of the bearing accompanied by the disappearance of the usual frequencies of the bearing and the appearance of random high frequency bands; in the same time, the frequency of rotation becomes dominant and the number of its higher harmonics increases.

The excitation harmonics are correlated with the frequencies that appear in the last stage and, consequently, many spectral components become visible, which hinders the interpretation of the measured signals.

The frequencies that are specific to the defects of the rolling bearings depend on the frequency of rotation of the shaft, $f_{r}=n / 60$, where $n$ is the shaft speed in rpm, without being integral multiples of it. It can be stated that the defects of the rolling bearings are among the few sources of vibrations that generate harmonics which are not multiples of the frequency of rotation. When in the spectrum no frequencies that are specific to defects appear, it means that no defects exist. On the contrary, the appearance of such frequencies should be regarded as an 
evidence of failure. These frequencies and the relationships that allow to calculate them are the following [5-7]:

- fundamental train frequency (for a defect on the cage or separator):

$$
F T F=f_{r} \frac{1}{2}\left(1-\frac{D_{b}}{D_{m}} \cos \beta\right) \quad[\mathrm{Hz}]
$$

- ball passing frequency inner race (for a defect on the inner race):

$$
B P F I=f_{r} \frac{Z}{2}\left(1+\frac{D_{b}}{D_{m}} \cos \beta\right) \quad[\mathrm{Hz}]
$$

- ball passing frequency outer race (for a defect on the outer race):

$$
B P F O=f_{r} \frac{Z}{2}\left(1-\frac{D_{b}}{D_{m}} \cos \beta\right) \quad[\mathrm{Hz}]
$$

- ball spin frequency:

$$
B S F=f_{r} \frac{D_{m}}{2 D_{b}}\left[1-\left(\frac{D_{b}}{D_{m}} \cos \beta\right)^{2}\right]
$$

- ball fault frequency (for a defect on a rolling element):

$$
B F F=2 B S F=f_{r} \frac{D_{m}}{D_{b}}\left[1-\left(\frac{D_{b}}{D_{m}} \cos \beta\right)^{2}\right] \quad[\mathrm{Hz}] .
$$

In the above formulas, $f_{r}$ is the frequency of rotation of the shaft, $Z$ is the number of rolling elements, that can be either balls or rollers, $\beta$ is the contact angle, $D_{m}$ is the pitch diameter (the average diameter of the bearing), and $D_{b}$ is the diameter of the rolling element.

The relationships presented above were used to calculate coefficients that multiply the frequency of rotation in order to obtain those frequencies that indicate a particular defect. Such coefficients are usually tabulated in catalogs provided by producers of rolling bearings [8].

\section{Experimental setups}

As mentioned in the Introduction, the vibrations were measured on two experimental models: one of a standard pump station and the other of a small hydropower plant.

The experimental setup of the pump station, schematically depicted in Figure 1, contains a free surface water tank, a suction pipe having the inner diameter of $128 \mathrm{~mm}$, a centrifugal

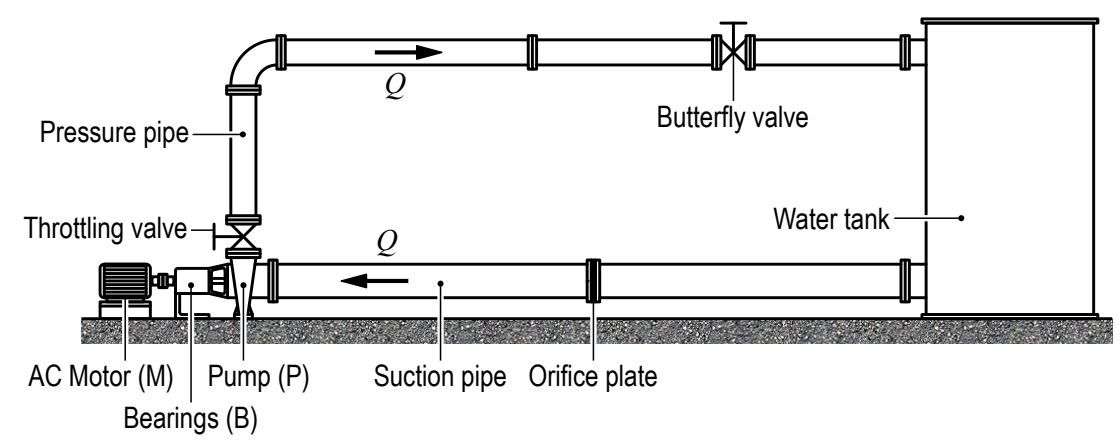

Fig. 1. Schematic of the experimental setup of the pump station. 
pump driven by a three-phase AC motor, and a discharge pipe having the inner diameter of $104 \mathrm{~mm}$. The AC motor has four poles, a rated power of $4 \mathrm{~kW}$, and a nominal speed of $1460 \mathrm{rpm}$, to which a frequency of rotation, $f_{r}$, of $24.3 \mathrm{~Hz}$ corresponds. Another frequency which is important for turbomachines, i.e. machines provided with a bladed impeller or runner, is the blade passing freqency, $f_{b}$, defined as follows:

$$
f_{b}=Z_{b} f_{r}
$$

where $Z_{b}$ is the number of impeller or runner blades. The pump impeller has 5 blades, hence the blade passing frequency is of $121.5 \mathrm{~Hz}$. The pump shaft is supported by two ball bearings of series 6310 and is connected to the motor shaft by a pin and bush coupling. At the pump outlet a gate valve for throttling the discharge is installed. The discharge is measured with an orifice plate installed in the suction pipe. The rather unusual installation of the orifice plate is due to the fact that this experimental setup can also be used to investigate the cavitation caused by a butterfly valve installed in the discharge pipe $[3,9]$. Both the throttling valve and the butterfly valve can be used to simulate the variable demand of consumers supplied with water by the pump. To measure the differential pressure caused by the orifice plate, an U-tube differential manometer with mercury was used. At zero head, the pump delivers a maximum flow rate, $Q_{\max }$, of roughly $43 \mathrm{l} / \mathrm{s}$. This approximate value was obtained by extrapolating the head curve obtained experimentally.

Not all the data required by the relationships (1) to (5) were available for the bearings of series 6310 . For this reason, the failing frequencies of these bearings were calculated by analogy with a similar bearing of series 310 S from the MRC catalog [8].

The experimental model of the small hydropower plant is schematically depicted in Figure 2. The water turbine is of Banki type and is fed with water by a pumping station through an upstream pipe. Under the experimental conditions, the maximum power that can

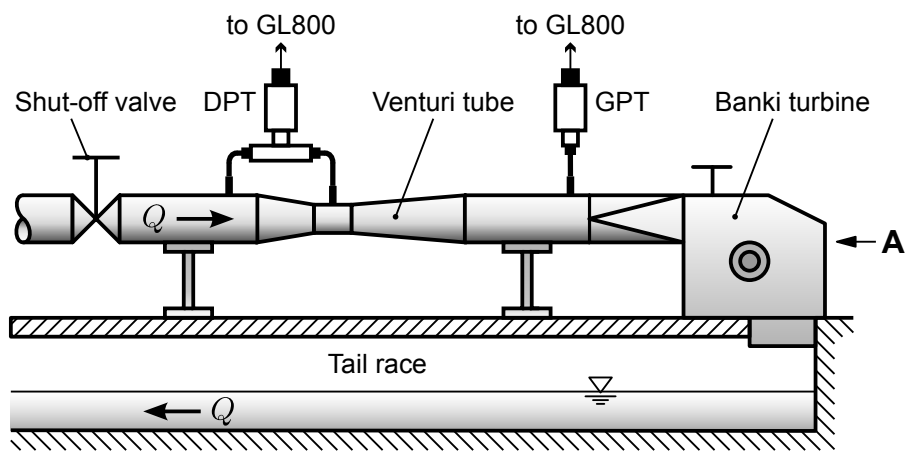

View from $A$

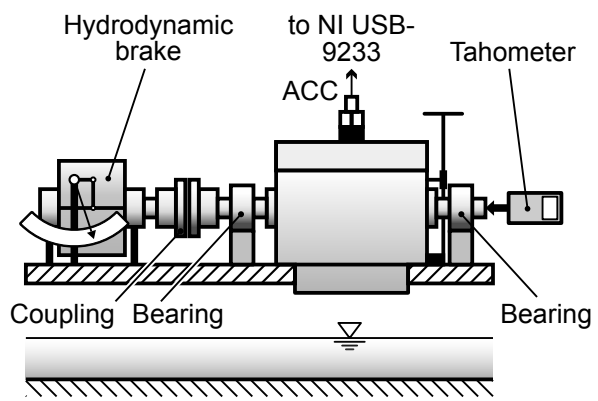

Data aquisition system

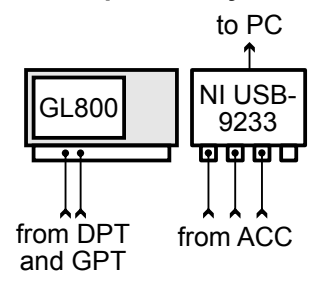

Fig. 2. Schematic of the experimental model of the small hydropower plant. 
be attained by the turbine is of roughly $1.1 \mathrm{~kW}$, although the turbine was initially designed for a higher power. The pipe has the inner diameter of $300 \mathrm{~mm}$ and is provided with a shut-off valve and with a welded Venturi tube for discharge measurement. The turbine is coupled by a pin and bush type coupling to its load represented by a hydrodynamic brake with oscillating stator, which replaces the generator. The brake allows to easily simulate different loads and to measure the corresponding torque (or shaft moment). It should be mentioned that, due to its operating principle, the hydrodynamic brake does not allow to slow down the turbine below a threshold speed, since the efficiency of the brake decreases rapidly as the shaft speed decreases. Nevertheless, the hydrodynamic brake has the important advantages of not generating additional shocks and vibrations and of allowing to accurately measure the torque.

The theory, construction and operation of Banki turbines are thoroughly described by Mockmore and Merryfield [10]. It should be mentioned here that these turbines are provided with an inner flap that allows the adjustment of the discharge. Banki water turbines are nowadays increasingly used in small hydro power plants due to their advantages, such as simple construction and maintenance, low price, and flat efficiency curve.

The runner of the tested turbine has 19 blades, a breadth of $300 \mathrm{~mm}$, and the outer and inner diameters of $250 \mathrm{~mm}$ and $165 \mathrm{~mm}$, respectively. The runner shaft sits on two double-row self-aligning radial ball bearings of series 2213 , having the following design parameters: $Z=18$ balls, $\beta=0^{\circ}, D_{m}=93 \mathrm{~mm}$, and $D_{b}=14 \mathrm{~mm}$.

The differential pressure at the Venturi tube and the static pressure at the turbine inlet were measured with a differential pressure transducer (DPT) and a gauge pressure transducer (GPT), respectively. The DPT and GPT have the measuring ranges of $0 \ldots 100 \mathrm{mbar}$ and $0 \ldots 2.5 \mathrm{bar}$, respectively. Both transducers have accuracies of $0.5 \%$ FS (i.e. of their measuring ranges). The transducers were connected to a GL800 data logger for data acquisition.

At both experimental setups, the vibrations levels were measured with three identical Brüel \& Kjaer DeltaTron accelerometers (denoted ACC in Figure 2) provided with built-in preamplifier and transducer data sheet (TEDS), that were connected to a laptop via a portable bus-powered USB carrier from National Instruments. The reason for using three accelerometers is that measurements were carried out simultaneously either at three locations or in three directions, as it will be detailed below. The accelerometers have the reference sensitivity of $20 \mathrm{~m} / \mathrm{s}^{2}$ RMS at $159.2 \mathrm{~Hz}$, the measuring range of $\pm 7000 \mathrm{~m} / \mathrm{s}^{2}$ peak, the frequency range $10 \%$ of $0.1 \mathrm{~Hz}$ to $6 \mathrm{kHz}$, and the phase response $\pm 5^{\circ}$ of $0.5 \mathrm{~Hz}$ to $5 \mathrm{kHz}$ [9].

At the pump, the vibrations levels were measured for the shaft speed of $1460 \mathrm{rpm}$ at zero flow rate (i.e. with the throttling valve fully closed) and at two flow rates close to the best efficiency point. For each flow rate the measurements were carried out simultaneously only in vertical direction on the motor, on the bearings housing and on the pump. The three measuring points will be denoted $\mathrm{M}, \mathrm{B}$, and $\mathrm{P}$, respectively. The sampling frequency was of $50 \mathrm{kHz}$. The high value of the sampling frequency was chosen in order to catch the effects of the hydrodynamic interaction inside the pump between the runner blades and the tongue of the scroll casing and of the pressure waves that could propagate back and forth between the pump and the partly opened gate (or disk) of the throttling valve.

At the turbine, the vibrations levels were measured for a flap opening $a_{0}$ of $25 \%$ of the maximum opening at four decreasing shaft speeds that were obtained by progressively filling the hydrodynamic brake with water: $448.8 \mathrm{rmp}, 397.7 \mathrm{rpm}, 322.8 \mathrm{rpm}$, and $294.8 \mathrm{rpm}$. Under the measurement conditions, the chosen flap opening allowed to obtain the highest overspeed and the largest speed interval. The measurements were carried out only on the turbine casing in three directions simultaneously: transverse direction, i.e. the direction of the upstream pipe that feeds the turbine, vertical direction, and axial direction, i.e. the direction of the turbine shaft axis. These three directions will be denoted $\mathrm{T}, \mathrm{V}$, and $\mathrm{A}$, respectively. The reason for measuring in three directions simultaneously was to identify the direction which better highlights the dominant 
frequencies of the vibrations. The sampling frequency was of $2 \mathrm{kHz}$. The value of the sampling frequency was chosen significantly lower than that used at the pump since no high frequency hydrodynamic phenomena was expected inside the turbine due to its operation at atmospheric pressure and only partly filled with water.

After the measurements, all data sets delivered by the accelerometers were filtered with a digital high-pass filter using the software dbTrait [11]. The cut-off frequency was chosen at $2 \mathrm{~Hz}$ to remove the low frequency random noise coming from outside of the experimental setups.

\section{Results and discussion}

The failing frequencies of the turbine bearings were calculated with the relationships (1) to (5). As mentioned previously, in the absence of some parameters, the failing frequencies of the pump bearings were calculated by analogy with corresponding bearings from the MRC catalog. The results along with the frequencies of rotation and the blade passing frequencies are presented in Table 1 . The calculated values were used to identify the failing frequencies in the frequency spectra of the signals obtained from the vibrations measurements.

The values of the failing frequencies obtained by analyzing the frequency spectra of the measured vibrations are presented in Table 2 together with the errors relative to the calculated values from Table 1 . In the table, exp. means experimental value, while err. means error relative to calculated value. It can be seen that all relative errors are below $5 \%$, which indicates that the theoretical and experimental failing frequencies are in good agreement.

The Figures 3 to 5 present FFT spectra of the vibrations measured at the pump - on the motor, on the bearings housing, and on the pump itself - for the three operating regimes described previously. The frequency of rotation, $f_{r}$, and its second and third-order harmonics, $2 f_{r}$ and $3 f_{r}$, respectively, as well as the blade passing frequency, $f_{b}$, can be easily identified.

Table 1. Speeds, frequencies of rotation, blade passing frequencies of the runners, and calculated failing frequencies of the bearings in the experimental setups.

\begin{tabular}{|cc|c|c|c|c|c|}
\hline \multicolumn{2}{|c|}{ Parameter } & Pump & \multicolumn{4}{c|}{ Banki turbine } \\
\hline$n$ & $(\mathrm{rpm})$ & 1460 & 448.8 & 397.7 & 322.8 & 294.8 \\
\hline$f_{r}$ & $(\mathrm{~Hz})$ & 24.33 & 7.48 & 6.63 & 5.38 & 4.91 \\
\hline$f_{b}$ & $(\mathrm{~Hz})$ & 121.7 & 142.1 & 126.0 & 102.2 & 93.29 \\
\hline$F T F$ & $(\mathrm{~Hz})$ & 9.27 & 3.18 & 2.82 & 2.28 & 2.09 \\
\hline$B F F=2 B S F$ & $(\mathrm{~Hz})$ & 96.38 & 49.68 & 44.02 & 35.73 & 32.63 \\
\hline$B P F I$ & $(\mathrm{~Hz})$ & 120.5 & 77.45 & 68.64 & 55.71 & 50.88 \\
\hline$B P F O$ & $(\mathrm{~Hz})$ & 74.15 & 57.19 & 50.67 & 41.13 & 37.56 \\
\hline
\end{tabular}

Table 2. Failing frequencies obtained experimentally for the investigated bearings.

\begin{tabular}{|c|c|c|c|c|c|c|c|c|c|c|}
\hline \multirow{3}{*}{ Parameter } & \multicolumn{2}{|c|}{ Pump } & \multicolumn{7}{|c|}{ Banki turbine } \\
\cline { 2 - 12 } & $n=1460 \mathrm{rpm}$ & $n=448.8 \mathrm{rpm}$ & \multicolumn{1}{|c|}{$n=397.7 \mathrm{rpm}$} & \multicolumn{2}{|c|}{$n=322.8 \mathrm{rpm}$} & \multicolumn{2}{c|}{$n=294.8 \mathrm{rpm}$} \\
\cline { 2 - 12 } & exp. & err. & exp. & err. & exp. & err. & exp. & err. & exp. & err. \\
\cline { 2 - 12 } & $(\mathrm{Hz})$ & $(\%)$ & $(\mathrm{Hz})$ & $(\%)$ & $(\mathrm{Hz})$ & $(\%)$ & $(\mathrm{Hz})$ & $(\%)$ & $(\mathrm{Hz})$ & $(\%)$ \\
\hline$f_{r}$ & 24.41 & 0.33 & 7.44 & 0.53 & 6.84 & 3.17 & 5.37 & 0.19 & 4.88 & 0.61 \\
\hline$F T F$ & 9.16 & 1.19 & 3.31 & 4.09 & 2.93 & 3.90 & 2.32 & 1.75 & 2.08 & 0.48 \\
\hline$B F F=2 B S F$ & 96.64 & 0.27 & 50.17 & 0.99 & 44.68 & 1.50 & 36.01 & 0.78 & 32.84 & 0.64 \\
\hline$B P F I$ & 121.05 & 0.46 & 77.15 & 0.39 & 68.6 & 0.06 & 55.54 & 0.31 & 50.42 & 0.90 \\
\hline$B P F O$ & 74.77 & 0.84 & 57.86 & 1.17 & 51.39 & 1.42 & 41.5 & 0.90 & 37.84 & 0.75 \\
\hline
\end{tabular}




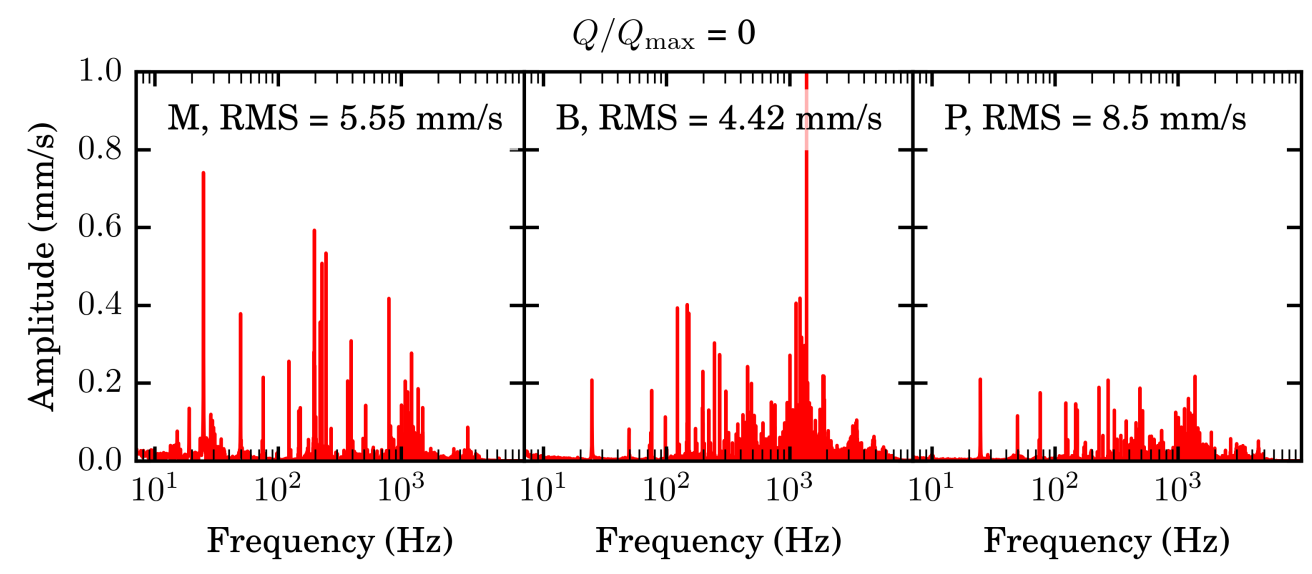

Fig. 3. FFT spectra of vibrations measured at the centrifugal pump at $Q / Q_{\max }=0$ (zero flow rate).

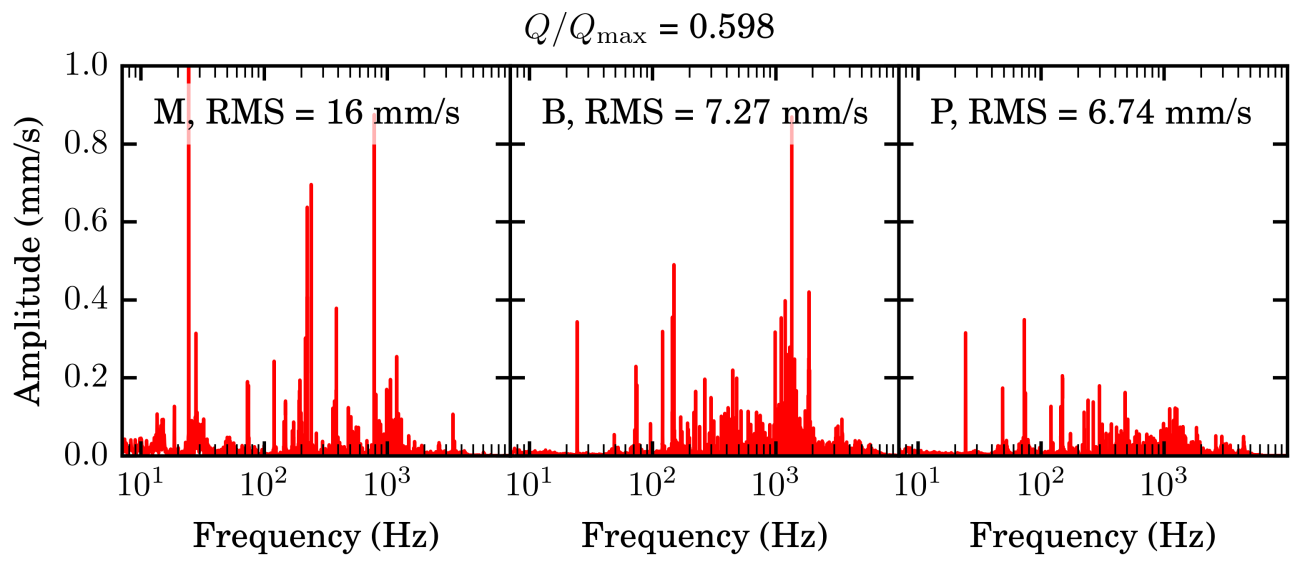

Fig. 4. FFT spectra of vibrations measured at the centrifugal pump at $Q / Q_{\max }=0.598$.

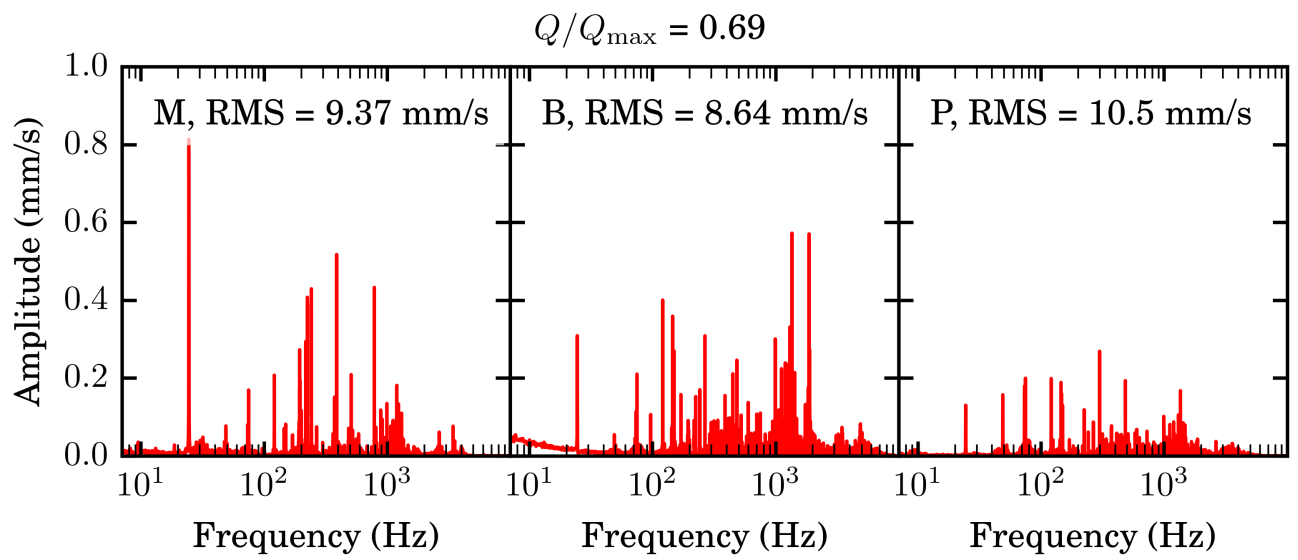

Fig. 5. FFT spectra of vibrations measured at the centrifugal pump at $Q / Q_{\max }=0.69$. 
In case of the motor, the frequency of rotation is always dominant in the spectra. On the pump, the amplitudes at $f_{r}$ are close to the maxima in the spectra. On the bearings, the amplitudes at $f_{r}$ are close to the average values in the spectra. The BPFI and at least its second-order harmonics (2BPFI) is also visible on all spectra - even at the motor. The same remark is valid for the $B P F O$ and its harmonics. It should be noted that, for the measurements on the bearings, the level of the spectral components associated to these frequencies is dominant in the specific frequency domain, i.e. below $500 \mathrm{~Hz}$. The same remark remains valid for the pump as well. This behavior is rather natural when seen in relation to the experimental assembly.

The analysis of the experimental data obtained at the Banki turbine for the four operating speeds (Figures 6 to 9) reveals that in all cases the FFT spectra of the vibrations measured in axial direction are more relevant than those corresponding to the transverse and vertical directions. This could be explained by the fact that the vibrations are dampened in transverse direction due to the rigid assembly of the turbine with the upstream pressure pipe, while in vertical direction the dampening is caused by the rigid mounting of the turbine on the concrete laboratory floor. Nevertheless, some important frequencies can also be observed on the spectra obtained for the transverse and vertical directions. Independent of the speed, dominant amplitudes at the frequencies of rotation, $f_{r}$, are clearly visible on all spectra. Only the first-order harmonic, $2 f_{r}$, can be clearly observed at the highest speed (Figure 6), while

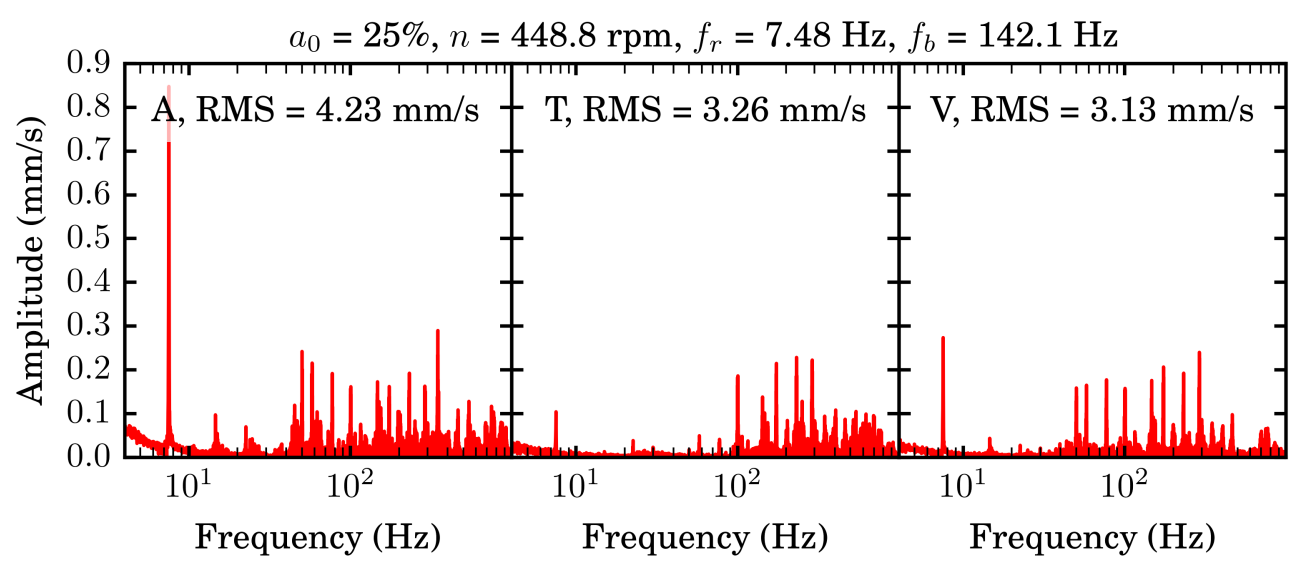

Fig. 6. FFT spectra of vibrations measured on the Banki turbine at $n=448.8 \mathrm{rpm}$.

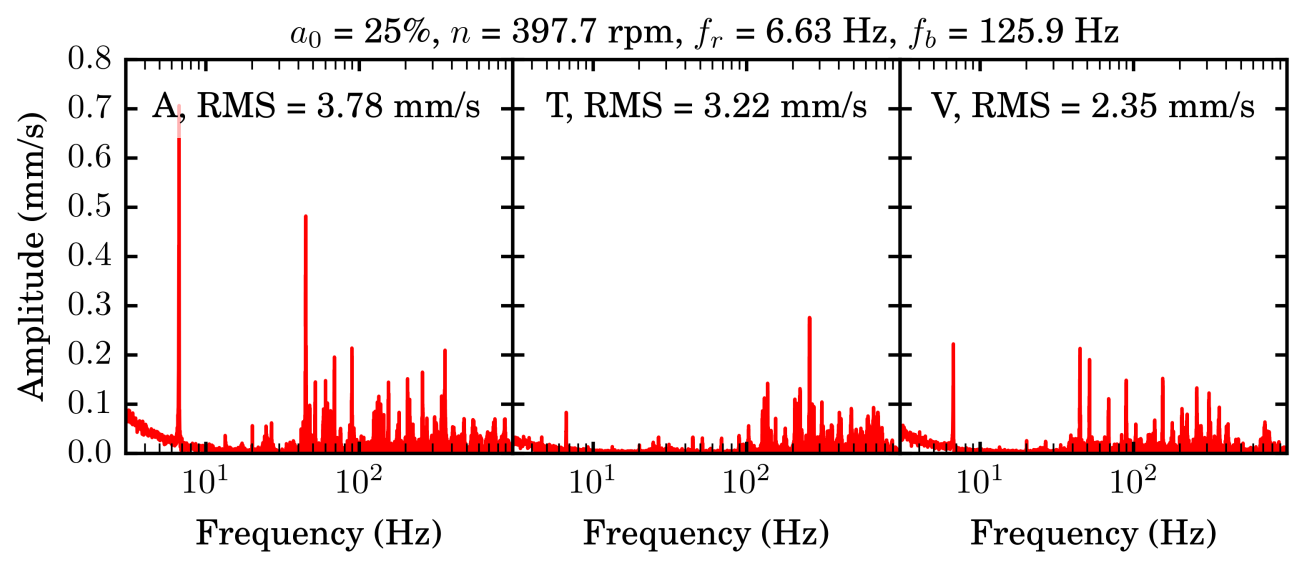

Fig. 7. FFT spectra of vibrations measured on the Banki turbine at $n=397.7 \mathrm{rpm}$. 


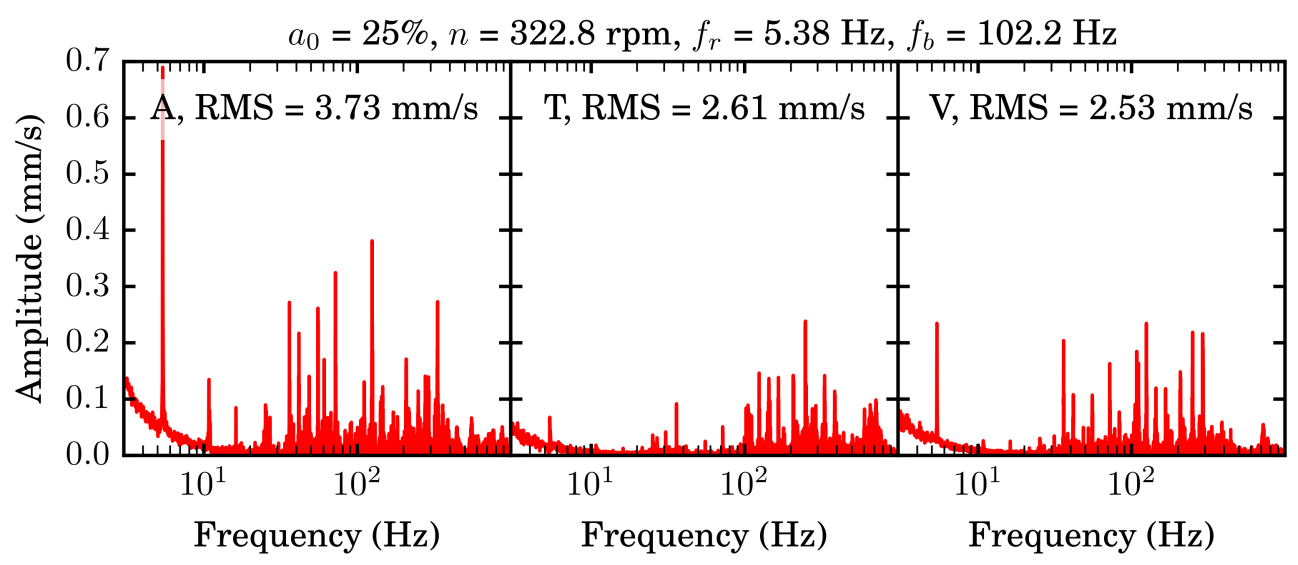

Fig. 8. FFT spectra of vibrations measured on the Banki turbine at $n=322.8 \mathrm{rpm}$.

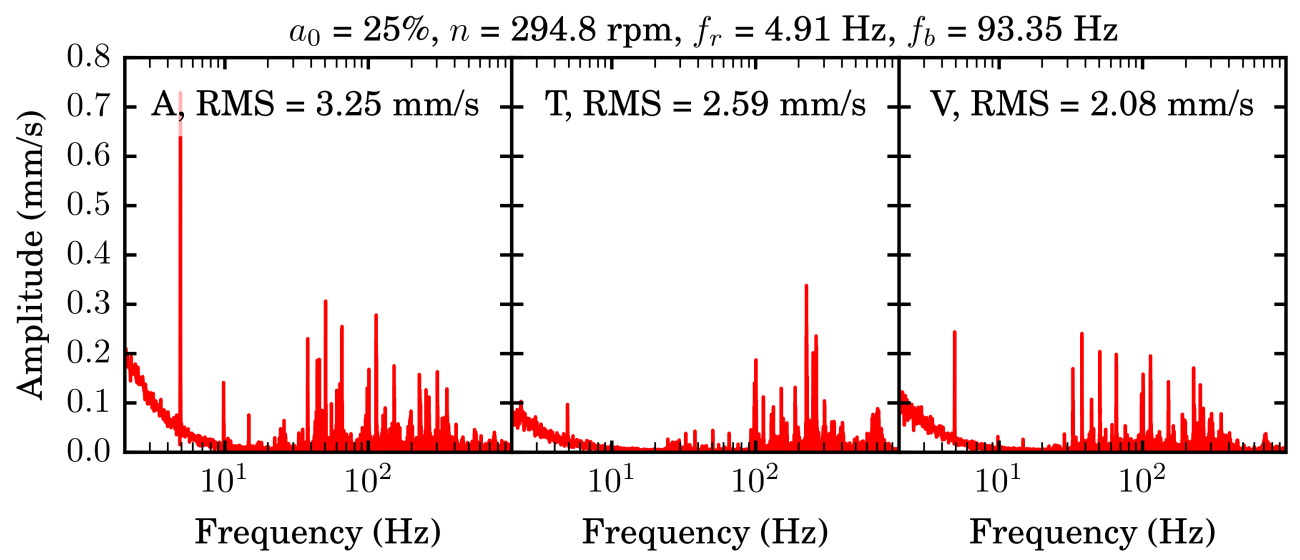

Fig. 9. FFT spectra of vibrations measured on the Banki turbine at $n=294.8 \mathrm{rpm}$.

two harmonics, at $2 f_{r}$ and $3 f_{r}$, are visible at the other speeds (Figures 9 to 7 ). It is interesting to note the the blade passing frequency can be easily identified at the maximum speed of $448.8 \mathrm{rpm}$ (Figure 6), although its corresponding amplitude is relatively low, and becomes less visible as the speed decreases (Figures 7 to 9). For all four speeds, significant peaks are visible at the corresponding BPFIs. The second and third order harmonics of the BPFIs are also visible. Peaks can be noticed at the $B P F O$ s and, in some cases, at their second-order harmonics, although they are smaller than those at the BPFIs. At higher speeds, local maxima are visible at the $B F F$ s (Figures 6 to 8). It should be noted, however, that all the measurements were carried out on the turbine casing, where other factors - mostly hydraulic phenomena could influence the vibrations levels as well.

\section{Conclusions}

In this paper, the vibrations measured on two experimental models of a pump station and of a small hydropower plant were analyzed in order to identify in their frequency spectra those frequencies that are specific to defects caused by the wear of the bearings.

The results obtained suggest that both the bearings of the pump and those of the turbine have defects on their inner and outer ring raceways. These defects are indicated by the occurrence of the corresponding failing frequencies and of their harmonics. 
In case of the pump, the ball fault frequency can also be identified, although it is very attenuated. Additionally, a high global RMS value can be noticed, both at the bearings housing and at the pump. Considering this high RMS value and the number of fault frequencies harmonics that can be identified in the spectra, it can be concluded that the pump bearings are affected by severe wear. According to the limit values for Small Machines of Class I, having powers below $15 \mathrm{~kW}$, from ISO 10816-1 [12], the bearings are at the upper limit of the Unsatisfactory (alert) zone, which has RMS values in the range $1.8-4.5 \mathrm{~mm} / \mathrm{s}$, and in the Unacceptable (danger) zone, characterized by RMS values above $4.5 \mathrm{~mm} / \mathrm{s}$. This indicates that the long-term operation of the pump should be restricted of even avoided, since the alarm values are exceeded and the shutdown values are almost attained.

In case of the Banki turbine, the occurrence of the ball fault frequency, even though not at all speeds, indicates the existence of an early failure of the rolling parts. In this case, the global RMS values place the turbine bearings in the Unsatisfactory (alert) zone according to ISO 10816-1 [12]. It should also be considered that the occurrence of the frequencies corresponding to defects of the raceways could indicate a poor lubrication or an incorrect loading of the bearings.

The results presented in this paper illustrate how tracing the fault frequencies in the spectra obtained during the operation of pumping systems or small water turbines and comparing the RMS values of the vibrations with those indicated by norms is an efficient method in monitoring the operation of such machines and in providing maintenance for them.

\section{References}

[1] E. Sima, Strategii Manageriale 4, 515 (2014)

[2] European Comission, Factsheet on 2014-2020 Rural Development Programme for Romania, (https://ec.europa.eu/agriculture/sites/agriculture/files/ rural-development-2014-2020/country-files/ro/factsheet_en.pdf)

[3] G. Băran, I. Catană, I. Magheți, C.A. Safta, M. Savu, IOP Conference Series Earth and Environmental Science 12, 012100 (2010)

[4] Y. Wu, S. Li, S. Liu, H.S. Dou, Z. Qian, Vibration of Hydraulic Machinery (Springer, Dordrecht, 2013)

[5] M. Gafitanu, S. Creu, B. Drăgan, Vibro-acoustic diagnostics of machines and equipment (Technical Publishing House, Bucharest, 1989)

[6] A. Bilosova, J. Bilos, Vibration Diagnostics, Investments in Education Development (Report CZ.1.07/2.2.00/15.0132, Ostrava, 2012)

[7] M. Rades, Dynamics of Machinery III (Printech, Bucharest, 2008)

[8] MRC Bearing Services, Engineering Handbook (SKF USA Inc., 2005)

[9] A. Dragomirescu, C.A. Safta, L. Mândrea, N. Orășanu, I. Magheți, Preventive Maintenance of Hydraulic Installations Based on Monitoring Cavitation-Induced Vibrations, in International Conference and Exposition on Electrical and Power Engineering (2018), pp. 883-888

[10] C.A. Mockmore, F. Merryfield, Oregon State College Engineering Experiment Station Bulletin 25 (1949)

[11] https://www.01db.com/activity-area/noise-and-vibration-monitoring/

[12] ISO 10816-1:1995 Mechanical vibration - Evaluation of machine vibrations by measurement on non-rotating parts - Part 1: General Guidelines (1995) 